\title{
On exact and approximate exchange-energy densities
}

\section{Springborg, Michael; Dahl, Jens Peder}

Published in:

Journal of Chemical Physics

Link to article, DOI:

$10.1063 / 1.478947$

Publication date:

1999

\section{Document Version}

Publisher's PDF, also known as Version of record

Link back to DTU Orbit

Citation (APA):

Springborg, M., \& Dahl, J. P. (1999). On exact and approximate exchange-energy densities. Journal of Chemical Physics, 110(19), 9360-9370. https://doi.org/10.1063/1.478947

\section{General rights}

Copyright and moral rights for the publications made accessible in the public portal are retained by the authors and/or other copyright owners and it is a condition of accessing publications that users recognise and abide by the legal requirements associated with these rights.

- Users may download and print one copy of any publication from the public portal for the purpose of private study or research.

- You may not further distribute the material or use it for any profit-making activity or commercial gain

- You may freely distribute the URL identifying the publication in the public portal

If you believe that this document breaches copyright please contact us providing details, and we will remove access to the work immediately and investigate your claim. 


\title{
On exact and approximate exchange-energy densities
}

\author{
Michael Springborg ${ }^{\text {a) }}$ \\ Department of Chemistry, University of Konstanz, D-78457 Konstanz, Germany ${ }^{\mathrm{b})}$ \\ and Department of Chemistry, Technical University of Denmark, DK-2800 Lyngby, Denmark \\ Jens Peder Dahlc) \\ Department of Chemistry, Technical University of Denmark, DK-2800 Lyngby, Denmark
}

(Received 21 December 1998; accepted 16 February 1999)

\begin{abstract}
Based on correspondence rules between quantum-mechanical operators and classical functions in phase space we construct exchange-energy densities in position space. Whereas these are not unique but depend on the chosen correspondence rule, the exchange potential is unique. We calculate this exchange-energy density for 15 closed-shell atoms, and compare it with kinetic- and Coulomb-energy densities. It is found that it has a dominating local-density character, but electron-shell effects are recognizable. The approximate exchange-energy functionals that have been proposed so far are found to account only poorly for the observed behaviors. Instead we use our results in proposing an alternative functional that depends on both first- and second-order derivatives of the electron density. (c) 1999 American Institute of Physics.
\end{abstract}

[S0021-9606(99)31218-6]

\section{INTRODUCTION}

During the last few decades density-functional methods have become an important tool in exploring electronic and structural properties of materials whereby all types of materials ranging from small molecules to infinite crystals currently are being treated (see, e.g., Ref. 1, and references therein). These methods are based on the theorems of Hohenberg and Kohn ${ }^{2}$ who showed that any ground-state property is a unique functional of the density of the system of interest. Kohn and $\mathrm{Sham}^{3}$ showed that it was useful to reformulate the problem of calculating the total electronic energy $E_{e}$ as that of solving a set of single-particle equations (in Hartree a.u.),

$$
\left[-\frac{1}{2} \nabla^{2}+V_{\text {eff }}(\mathbf{r})\right] \psi_{i}(\mathbf{r})=\epsilon_{i} \psi_{i}(\mathbf{r}) .
$$

$V_{\text {eff }}$ is an effective potential containing three terms,

$$
V_{\text {eff }}(\mathbf{r})=V_{n}(\mathbf{r})+V_{C}(\mathbf{r})+V_{\mathrm{xc}}(\mathbf{r}),
$$

where the first term is the Coulomb potential due to the nuclei, the second term is that of the electrons, and the last term on the right-hand side is the so-called exchangecorrelation potential.

$V_{\mathrm{xc}}(\mathbf{r})$ is defined as the functional derivative of the exchange-correlation energy,

$$
V_{\mathrm{xc}}(\mathbf{r})=\frac{\delta E_{\mathrm{xc}}}{\delta \rho(\mathbf{r})},
$$

with $E_{\mathrm{xc}}$ in turn being defined as a sum of the exchange and correlation energy

$$
E_{\mathrm{xc}}=-E_{\mathrm{x}}+E_{\mathrm{c}}
$$

\footnotetext{
${ }^{a)}$ Electronic mail: mcs@chclu.chemie.uni-konstanz.de

${ }^{b)}$ Permanent address.

${ }^{c)}$ Electronic mail: jpd@kemi.dtu.dk
}

Due to convenience, we have changed the sign of $E_{\mathrm{x}}$ compared to common practice.

Similar to $E_{e}, E_{\mathrm{x}}$ and $E_{\mathrm{c}}$ are functionals of $\rho(\mathbf{r})$ although their precise forms are unknown. In practical calculations one applies therefore approximations, which, e.g., for the exchange energy amounts to writing

$$
-E_{\mathrm{x}} \simeq \int \varepsilon_{\mathrm{x}}(\mathbf{r}) \cdot \rho(\mathbf{r}) d \mathbf{r},
$$

with $\varepsilon_{\mathrm{x}}$ at the point $\mathbf{r}$ being a function of the electron density and of some of its derivatives at this point,

$$
\varepsilon_{\mathrm{x}}(\mathbf{r})=\varepsilon_{\mathrm{x}}\left[\rho(\mathbf{r}),|\nabla \rho(\mathbf{r})|, \nabla^{2} \rho(\mathbf{r}), \ldots\right] .
$$

There exists a number of different approximations of this type and below we shall discuss some of those. For the present purpose it suffices to notice that $\varepsilon_{\mathrm{x}}(\mathbf{r}) \cdot \rho(\mathbf{r})$ of Eq. (5) plays the role of an exchange-energy density in position space.

A common approach for obtaining approximate functionals is to consider the total exchange energy, Eq. (5), for a set of systems and from this set of data try to derive the functional of Eq. (6). It should be obvious that this procedure is not unique, and that many different functionals may lead to similar results for the chosen set of systems. Alternatively, more detailed information is obtained by focusing on the exchange potential $V_{\mathrm{x}}(\mathbf{r})$, but despite the relation (3), it is in general not possible to extract the energy density of Eq. (5) uniquely from this information.

For closed-shell systems the Hartree-Fock approximation provides a useful alternative to the density-functional methods. In that case the exchange energy is defined as

$E_{\mathrm{x}}=2 \sum_{i, j=1}^{N / 2} \iint \frac{\phi_{i}^{*}\left(\mathbf{r}_{1}\right) \phi_{j}^{*}\left(\mathbf{r}_{2}\right) \phi_{j}\left(\mathbf{r}_{1}\right) \phi_{i}\left(\mathbf{r}_{2}\right)}{\left|\mathbf{r}_{1}-\mathbf{r}_{2}\right|} d \mathbf{r}_{1} d \mathbf{r}_{2}$, 
where we have assumed that the number of electrons $N$ is even and that we have $N / 2$ doubly degenerate, filled orbitals that pairwise only differ in the spin component.

Unfortunately, Eq. (7) does not directly lead to a unique definition of an exchange-energy density in position space. Any transformation of the form

$$
\left(\begin{array}{c}
\mathbf{r} \\
\mathbf{r}^{\prime}
\end{array}\right)=\mathrm{U} \cdot\left(\begin{array}{l}
\mathbf{r}_{1} \\
\mathbf{r}_{2}
\end{array}\right)
$$

and subsequently integration over $\mathbf{r}^{\prime}$ leads to a possible exchange-energy density. In Eq. (8), $U$ is any real matrix whose determinant equals \pm 1 . Studies for $U$ being the unit matrix were recently presented by Filippi, Gonze, and Umrigar, ${ }^{4}$ by Gritsenko, van Leeuwen, and Baerends, ${ }^{5}$ and by Hood et al. ${ }^{6}$

It is the purpose of the present work to show how the concepts of correspondence rules and phase-space functions can be used in obtaining exchange-energy densities in position space. This will be the subject of Sec. II. In Sec. III we shall show that independent of the chosen correspondence rule, the resulting exchange potential $V_{\mathrm{x}}$ is unique, although the exchange-energy density is not. The Weyl correspondence rule and the therewith connected Wigner phase-space function have certain conceptual advantages (to be discussed below) and therefore we shall in Sec. IV use those in specifying one particular exchange-energy density. Section $\mathrm{V}$ is devoted to results for 15 closed-shell atoms ( $\mathrm{He}, \mathrm{Be}, \mathrm{Ne}, \mathrm{Mg}$, $\mathrm{Ar}, \mathrm{Ca}, \mathrm{Zn}, \mathrm{Kr}, \mathrm{Sr}, \mathrm{Cd}, \mathrm{Xe}, \mathrm{Ba}, \mathrm{Yb}, \mathrm{Hg}$, and $\mathrm{Rn}$ ), and in Sec. VI we shall use the information thereby obtained in exploring the quality of a number of proposed approximate forms for the exchange-energy density. It is found that the approximate forms do not describe our results as accurately as might be desirable. Therefore, in Sec. VII we shall use our results in proposing another approximate form, and, finally, in Sec. VIII we offer a conclusion.

To our knowledge there exist only two previous studies where the exchange energy was studied from a phase-space point of view. In the first of those, ${ }^{7}$ some of the formulas that we shall discuss here were presented, and exchange-energy densities for some few closed-shell atoms were presented. But the attempts for constructing improved approximate density functionals were very limited. A somewhat different approach was taken by Ghosh and Parr ${ }^{8}$ who also used their results in proposing an approximate exchange-energy functional. They used, however, approximate phase-space functions whereby some uncontrolled approximations may have been introduced. We finally add that Burke, Perdew, and co-workers ${ }^{9-13}$ also have studied the total exchange energy by analyzing the energy density in position space. They used, however, a very different approach than ours, and also their emphasis is different from ours.

\section{CORRESPONDENCE RULES AND PHASE-SPACE FUNCTIONS}

For the sake of simplicity we consider a single-particle system. According to classical mechanics any quantity $A$ is a function in phase space. We may Fourier transform it,

$$
A(\mathbf{r}, \mathbf{p})=\frac{1}{(2 \pi)^{3}} \iint a(\mathbf{s}, \mathbf{t}) e^{i(\mathbf{r} \cdot \mathbf{s}+\mathbf{p} \cdot \mathbf{t})} d \mathbf{s} d \mathbf{t}
$$

with

$$
a(\mathbf{s}, \mathbf{t})=\frac{1}{(2 \pi)^{3}} \iint A(\mathbf{r}, \mathbf{p}) e^{-i(\mathbf{r} \cdot \mathbf{s}+\mathbf{p} \cdot \mathbf{t})} d \mathbf{r} d \mathbf{p} .
$$

The quantum-mechanical operator corresponding to $A$ (characterizing it by a caret) is then defined through

$$
\hat{A}=\frac{1}{(2 \pi)^{3}} \iint a(\mathbf{s}, \mathbf{t}) e^{i(\hat{\mathbf{r}} \cdot \mathbf{s}+\hat{\mathbf{p}} \cdot \mathbf{t}} g(\mathbf{s}, \mathbf{t}) d \mathbf{s} d \mathbf{t},
$$

where different correspondence rules correspond to different choices of $g(\mathbf{s}, \mathbf{t})$ (see, e.g., Refs. 14-16). $g(\mathbf{s}, \mathbf{t})$ is, in principle, general but is chosen to fulfill

$$
g(\mathbf{0 , t})=g(\mathbf{s}, \mathbf{0})=1 .
$$

It shall be stressed that there is no restrictive criterion that allows a unique specification of $g(\mathbf{s}, \mathbf{t})$. The Weyl correspondence, which is the one we shall pursue [see the discussion following Eq. (21)], has $g(\mathbf{s}, \mathbf{t})=1$.

Combining Eqs. (9) and (11) leads to

$$
\begin{aligned}
\hat{A}= & \iint A(\mathbf{r}, \mathbf{p}) \\
& \times\left[\frac{1}{(2 \pi)^{6}} \iint e^{-i(\mathbf{r} \cdot \mathbf{s}+\mathbf{p} \cdot \mathbf{t})} e^{i(\hat{\mathbf{r}} \cdot \mathbf{s}+\hat{\mathbf{p}} \cdot \mathbf{t})} g(\mathbf{s}, \mathbf{t}) d \mathbf{s} d \mathbf{t}\right] d \mathbf{r} d \mathbf{p} .
\end{aligned}
$$

This gives the prescription for constructing the quantummechanical operator from a given classical function. In order to obtain the reverse transformation we proceed as follows. We consider any two eigenstates of the position operator, $\left|\mathbf{r}^{\prime}\right\rangle$ and $\left|\mathbf{r}^{\prime \prime}\right\rangle$ and construct the matrix element for those with the operator of Eq. (13),

$$
\begin{aligned}
\left\langle\mathbf{r}^{\prime}|\hat{A}| \mathbf{r}^{\prime \prime}\right\rangle= & \iint A(\mathbf{r}, \mathbf{p})\left[\frac{1}{(2 \pi)^{6}} \iint e^{-i(\mathbf{r} \cdot \mathbf{s}+\mathbf{p} \cdot \mathbf{t})}\right. \\
& \left.\times\left\langle\mathbf{r}^{\prime}\left|e^{i(\hat{\mathbf{r}} \cdot \mathbf{s}+\hat{\mathbf{p}} \cdot \mathbf{t})}\right| \mathbf{r}^{\prime \prime}\right\rangle g(\mathbf{s}, \mathbf{t}) d \mathbf{s} d \mathbf{t}\right] d \mathbf{r} d \mathbf{p} .
\end{aligned}
$$

By applying the following identities: ${ }^{17}$

$$
\begin{aligned}
& e^{i(\hat{\mathbf{r}} \cdot \mathbf{s}+\hat{\mathbf{p}} \cdot \mathbf{t})}=e^{i(1 / 2) \mathbf{s} \cdot \mathbf{t}} e^{i \hat{\mathbf{r}} \cdot \mathbf{s}} e^{i \hat{\mathbf{p}} \cdot \mathbf{t}}, \\
& e^{i \hat{\mathbf{p}} \cdot \mathbf{t}}\left|\mathbf{r}^{\prime \prime}\right\rangle=\left|\mathbf{r}^{\prime \prime}-\mathbf{t}\right\rangle, \\
& e^{i \hat{\mathbf{r}} \cdot \mathbf{s}}\left|\mathbf{r}^{\prime \prime}-\mathbf{t}\right\rangle=e^{i\left(\mathbf{r}^{\prime \prime}-\mathbf{t}\right) \cdot \mathbf{s}}\left|\mathbf{r}^{\prime \prime}-\mathbf{t}\right\rangle, \\
& \left(\mathbf{r}^{\prime} \mid \mathbf{r}^{\prime \prime}-\mathbf{t}\right)=\delta\left(\mathbf{r}^{\prime}-\mathbf{r}^{\prime \prime}+\mathbf{t}\right),
\end{aligned}
$$

and setting

$$
\begin{aligned}
& \mathbf{r}^{\prime}=\mathbf{R}-\frac{1}{2} \mathbf{u}, \\
& \mathbf{r}^{\prime \prime}=\mathbf{R}+\frac{1}{2} \mathbf{u},
\end{aligned}
$$

we obtain after some straightforward manipulations 


$$
\begin{aligned}
\left\langle\mathbf{R}-\frac{1}{2} \mathbf{u}|\hat{A}| \mathbf{R}+\frac{1}{2} \mathbf{u}\right\rangle= & \iint A(\mathbf{r}, \mathbf{p}) \frac{1}{(2 \pi)^{6}} \\
& \times \int e^{i[\mathbf{s} \cdot(\mathbf{R}-\mathbf{r})-\mathbf{p} \cdot \mathbf{u}]} g(\mathbf{s}, \mathbf{u}) d \mathbf{s} d \mathbf{r} d \mathbf{p} .
\end{aligned}
$$

We multiply by $e^{-i \mathbf{q} \cdot \mathbf{R}}$ and integrate over $\mathbf{R}$ which gives

$$
\begin{aligned}
& \int\left\langle\mathbf{R}-\frac{1}{2} \mathbf{u}|\hat{A}| \mathbf{R}+\frac{1}{2} \mathbf{u}\right\rangle e^{-\mathbf{i q} \cdot \mathbf{R}} d \mathbf{R} \\
& =\frac{1}{(2 \pi)^{3}} \iint A(\mathbf{r}, \mathbf{p}) g(\mathbf{q}, \mathbf{u}) e^{-i(\mathbf{q} \cdot \mathbf{r}+\mathbf{p} \cdot \mathbf{u})} d \mathbf{r} d \mathbf{p},
\end{aligned}
$$

or, by inverting this Fourier transform,

$$
\begin{aligned}
A(\mathbf{r}, \mathbf{R})= & \frac{1}{(2 \pi)^{3}} \iiint \frac{1}{g(\mathbf{q}, \mathbf{u})}\left\langle\mathbf{R}-\frac{1}{2} \mathbf{u}|\hat{A}| \mathbf{R}+\frac{1}{2} \mathbf{u}\right\rangle \\
& \times e^{i(\mathbf{q} \cdot \mathbf{r}+\mathbf{p} \cdot \mathbf{u})} e^{-i \mathbf{q} \cdot \mathbf{R}} d \mathbf{R} d \mathbf{q} d \mathbf{u} .
\end{aligned}
$$

Equation (19) is the one we shall use in defining a function in phase space corresponding to the quantummechanical exchange operator. It is seen that this function will depend on the chosen correspondence rule through the function $g(\mathbf{q}, \mathbf{u})$ but the resulting exchange potential is nevertheless independent of the correspondence rule.

However, before discussing the exchange energies we shall define position-space densities in the general case. To this end we return to Eq. (13). Then, any matrix element $\left\langle\psi_{i}|\hat{A}| \psi_{j}\right\rangle$ can be expressed as

$$
\begin{aligned}
\left\langle\psi_{i}|\hat{A}| \psi_{j}\right\rangle= & \iint A(\mathbf{r}, \mathbf{p})\left[\frac{1}{(2 \pi)^{6}} \iint e^{-i(\mathbf{r} \cdot \mathbf{s}+\mathbf{p} \cdot \mathbf{t})}\right. \\
& \left.\times\left\langle\psi_{i}\left|e^{i(\hat{\mathbf{r}} \cdot \mathbf{s}+\hat{\mathbf{p}} \cdot \mathbf{t})}\right| \psi_{j}\right\rangle g(\mathbf{s}, \mathbf{t}) d \mathbf{s} d \mathbf{t}\right] d \mathbf{r} d \mathbf{p} \\
\equiv & \iint A(\mathbf{r}, \mathbf{p}) f_{i j}(\mathbf{r}, \mathbf{p}) d \mathbf{r} d \mathbf{p} .
\end{aligned}
$$

Here, $f_{i j}(\mathbf{r}, \mathbf{p})$ is a function in phase space that is independent of the operator and only depends on the two states $\psi_{i}$ and $\psi_{j}$ as well as on the chosen correspondence rule, characterized through the function $g(\mathbf{s}, \mathbf{t})$.

For $i=j$, the phase-space function $f_{i i}$ is that of a state, otherwise it is that of a transition. For $i=j$ it shares many properties with a probability distribution in phase space. For example, besides Eq. (20) we also have

$$
\begin{gathered}
\int f_{i i}(\mathbf{r}, \mathbf{p}) d \mathbf{p}=\left|\psi_{i}(\mathbf{r})\right|^{2}, \\
\int f_{i i}(\mathbf{r}, \mathbf{p}) d \mathbf{r}=\left|\psi_{i}(\mathbf{p})\right|^{2},
\end{gathered}
$$

which follows from Eq. (12). On the other hand, $f_{i i}(\mathbf{r}, \mathbf{p})$ is in general not real or non-negative, and only for the Weyl correspondence [in which case $g(\mathbf{s}, \mathbf{t}) \equiv 1$ and $f$ becomes Wigner's phase-space function $\left.{ }^{18}\right]$ is the phase-space function in the general case real but may be negative. This property together with some other useful properties of the Weyl correspondence (i.e., the fact that no other correspondence rule leads to operators that are invariant under certain canonical transformations, and the fact that the exchange hole as defined with the Weyl correspondence can be considered local) and of the Wigner function ${ }^{15,19,20}$ make us propose to base the discussion on the Wigner function and the Weyl correspondence rule.

Independent of the correspondence rule, Eq. (20) may be rewritten as

$\left\langle\psi_{i}|\hat{A}| \psi_{j}\right\rangle=\int\left[\int A(\mathbf{r}, \mathbf{p}) f_{i j}(\mathbf{r}, \mathbf{p}) d \mathbf{p}\right] d \mathbf{r} \equiv \int a_{i j}(\mathbf{r}) d \mathbf{r}$,

where $a_{i j}(\mathbf{r})$ is a position-space density for the quantummechanical operator and for the transition $(i \neq j)$ or state $(i=j)$ of interest.

\section{EXCHANGE-ENERGY DENSITIES AND POTENTIALS}

Equation (7) can be written as a sum of expectation values for the single-particle orbitals,

$$
E_{\mathrm{x}}=2 \sum_{i, j=1}^{N / 2}\left\langle\phi_{i}\left|\hat{K}_{j}\right| \phi_{i}\right\rangle,
$$

where the exchange operator $\hat{K}_{j}$ for the $j$ th orbital is defined as

$$
\hat{K}_{j}=\hat{K}_{j}(\mathbf{r})=\int \frac{\phi_{j}^{*}\left(\mathbf{r}^{\prime}\right) \hat{P}_{12} \phi_{j}\left(\mathbf{r}^{\prime}\right)}{\left|\mathbf{r}-\mathbf{r}^{\prime}\right|} d \mathbf{r}^{\prime} .
$$

Here, $\hat{P}_{12}$ is a permutation operator that interchanges the arguments of the following two functions.

Equation (19) makes it possible to define a function in phase space according to the operator of Eq. (24). To this end we need

$$
\left\langle\mathbf{R}-\frac{1}{2} \mathbf{u}\left|\hat{K}_{j}\right| \mathbf{R}+\frac{1}{2} \mathbf{u}\right\rangle=\frac{\phi_{j}^{*}\left(\mathbf{R}-\frac{1}{2} \mathbf{u}\right) \phi_{j}\left(\mathbf{R}+\frac{1}{2} \mathbf{u}\right)}{|\mathbf{u}|},
$$

giving

$$
\begin{aligned}
K_{j}(\mathbf{r}, \mathbf{p})= & \frac{1}{(2 \pi)^{3}} \iiint \frac{1}{g(\mathbf{q}, \mathbf{u})} e^{i \mathbf{q} \cdot(\mathbf{r}-\mathbf{R})} \\
& \times e^{i \mathbf{p} \cdot \mathbf{u}} \frac{\phi_{j}^{*}\left(\mathbf{R}-\frac{1}{2} \mathbf{u}\right) \phi_{j}\left(\mathbf{R}+\frac{1}{2} \mathbf{u}\right)}{u} d \mathbf{R} d \mathbf{q} d \mathbf{u} .
\end{aligned}
$$

This is the "classical" function in phase space corresponding to the exchange operator $\hat{K}_{j}$ of Eq. (24).

Equation (26) leads to the following exchange-energy density in position space:

$$
\boldsymbol{\epsilon}_{\mathrm{x}}(\mathbf{r})=2 \sum_{i, j=1}^{N / 2} \int K_{j}(\mathbf{r}, \mathbf{p}) f_{i i}(\mathbf{r}, \mathbf{p}) d \mathbf{p}
$$

[notice, $\epsilon_{\mathrm{x}}$ of Eq. (27) is not the same as $\varepsilon_{\mathrm{x}}$ of Eq. (5)], and the total exchange energy is obtained by integration $\epsilon_{\mathrm{x}}(\mathbf{r})$ over the complete space,

$$
E_{\mathrm{x}}=\int \epsilon_{\mathrm{x}}(\mathbf{r}) d \mathbf{r} \text {. }
$$

Also Ghosh and $\operatorname{Parr}^{8}$ calculated an exchange-energy density in position space by considering a phase-space den- 
sity based on Wigner's phase-space functions. They, however, approximated the latter by a functional of the electron density and the kinetic-energy density, whereby they obtained approximate exchange-energy densities. For the kinetic-energy density they used actually the form given below [Eq. (34)] without noticing its relation to Wigner's phase-space function.

Whereas $\epsilon_{\mathrm{x}}(\mathbf{r})$ depends on the correspondence rule, $E_{\mathrm{x}}$ does not. Furthermore, therefore also the exchange potential

$$
V_{\mathrm{x}}(\mathbf{r})=\frac{\delta E_{\mathrm{x}}}{\delta \rho(\mathbf{r})}
$$

is independent of the correspondence rule.

\section{THE EXCHANGE-ENERGY DENSITY FOR THE WEYL CORRESPONDENCE}

As discussed in Sec. II, the Weyl correspondence and the thereto related Wigner phase-space function have particularly appealing properties that suggest basing the discussion on those. Then, we set

$$
g(\mathbf{s}, \mathbf{t})=1,
$$

and after some straightforward manipulation we obtain ${ }^{7}$

$$
\begin{aligned}
\boldsymbol{\epsilon}_{\mathrm{x}}(\mathbf{r})= & 8 \sum_{i, j=1}^{N / 2} \int \frac{\phi_{i}^{*}\left(\mathbf{r}-\mathbf{r}_{1}\right) \phi_{i}\left(\mathbf{r}+\mathbf{r}_{1}\right) \phi_{j}^{*}\left(\mathbf{r}+\mathbf{r}_{1}\right) \phi_{j}\left(\mathbf{r}-\mathbf{r}_{1}\right)}{r_{1}} \\
& \times d \mathbf{r}_{1} .
\end{aligned}
$$

Comparing with Eqs. (7) and (8) we see that this corresponds to one particular choice for the matrix $U$,

$$
U=\left(\begin{array}{cc}
\frac{1}{2} & \frac{1}{2} \\
-1 & 1
\end{array}\right)
$$

We shall compare the exchange-energy density with the Coulomb and the kinetic-energy densities that can be defined following a similar procedure. For the former we obtain

$$
\epsilon_{C}(\mathbf{r})=2 \sum_{i, j=1}^{N / 2} \int \frac{\phi_{i}^{*}\left(\mathbf{r}_{1}-\mathbf{r}\right) \phi_{i}\left(\mathbf{r}_{1}-\mathbf{r}\right) \phi_{j}^{*}(\mathbf{r}) \phi_{j}(\mathbf{r})}{r_{1}} d \mathbf{r}_{1},
$$

whereas the kinetic-energy density is given through ${ }^{7,21}$

$$
\begin{aligned}
\epsilon_{\mathrm{k}}(\mathbf{r})= & 2 \sum_{i=1}^{N / 2}\left[-\frac{1}{8} \phi_{i}^{*}(\mathbf{r}) \nabla^{2} \phi_{i}(\mathbf{r})-\frac{1}{8} \phi_{i}(\mathbf{r}) \nabla^{2} \phi_{i}^{*}(\mathbf{r})\right. \\
& \left.+\frac{1}{4}\left|\nabla \phi_{i}(\mathbf{r})\right|^{2}\right] .
\end{aligned}
$$

The latter is obtained using a procedure analogous to that for the exchange-energy density, i.e., from the classical expression for the kinetic energy in phase space, $\left(p^{2} / 2\right)$, we calculate the kinetic-energy density in phase space and through integration over the momentum coordinates we obtain the density in position space.

\section{RESULTS FOR CLOSED-SHELL ATOMS}

We have calculated the various energy densities for the 15 closed-shell atoms $\mathrm{He}, \mathrm{Be}, \mathrm{Ne}, \mathrm{Mg}, \mathrm{Ar}, \mathrm{Ca}, \mathrm{Zn}, \mathrm{Kr}, \mathrm{Sr}$, $\mathrm{Cd}, \mathrm{Xe}, \mathrm{Ba}, \mathrm{Yb}, \mathrm{Hg}$, and $\mathrm{Rn}$. Due to the closed shells the Hartree-Fock approximation is a good one.

The orbitals can be characterized by the three quantum numbers $(n, l, m)$ (neglecting spin),

$$
\phi_{i}(\mathbf{r})=\phi_{n l m}(\mathbf{r})=R_{n l}(r) Y_{l m}(\theta, \phi),
$$

where $R_{n l}(r)$ is expanded in a series of Gaussians as given by Huzinaga and Klobukowski. ${ }^{22}$

Ultimately we shall be interested in relating our results to density-functional calculations, and it may therefore be questioned whether it is a reasonable approximation to replace the orbitals of Eq. (35) by the Hartree-Fock orbitals of Huzinaga and Klobukowski. ${ }^{22}$ However, a comparison (not shown) between the functions of Huzinaga and Klobukowski and those of a density-functional calculation (with the localdensity approximation of von Barth and $\operatorname{Hedin}^{23}$ for exchange and correlation effects) for the isolated atoms shows that the two sets of functions in fact are very similar except for the outermost regions of the atoms, where the densityfunctional densities tend to fall off more rapidly than the Hartree-Fock densities. This may be considered related to the problem that the currently applied local-density approximations do not have the proper asymptotic behaviors.

For the closed-shell atoms the exchange-energy density becomes

$$
\begin{aligned}
\epsilon_{\mathbf{x}}(\mathbf{r})= & 8 \sum_{n_{1}, n_{2}} \sum_{l_{1}, l_{2}} \sum_{m_{1}, m_{2}} \int \frac{1}{r_{1}} \phi_{n_{1}, l_{1}, m_{1}}^{*}\left(\mathbf{r}-\mathbf{r}_{1}\right) \\
& \times \phi_{n_{1}, l_{1}, m_{1}}\left(\mathbf{r}+\mathbf{r}_{1}\right) \phi_{n_{2}, l_{2}, m_{2}}^{*}\left(\mathbf{r}+\mathbf{r}_{1}\right) \\
& \times \phi_{n_{2}, l_{2}, m_{2}}\left(\mathbf{r}-\mathbf{r}_{1}\right) d \mathbf{r}_{1} \\
= & 8 \sum_{n_{1}, n_{2}} \sum_{l_{1}, l_{2}} \sum_{m_{1}, m_{2}}(-1)^{l_{1}+l_{2}} \int \frac{1}{r_{1}} R_{n_{1}, l_{1}}^{*}\left(r_{a}\right) \\
& \times R_{n_{2}, l_{2}}\left(r_{a}\right) R_{n_{1}, l_{1}}\left(r_{b}\right) R_{n_{2}, l_{2}}^{*}\left(r_{b}\right) \\
& \times Y_{l_{1}, m_{1}}^{*}\left(\theta_{a}, \phi_{a}\right) Y_{l_{2}, m_{2}}\left(\theta_{a}, \phi_{a}\right) Y_{l_{1}, m_{1}}\left(\theta_{b}, \phi_{b}\right) \\
& \times Y_{l_{2}, m_{2}}^{*}\left(\theta_{b}, \phi_{b}\right) d \mathbf{r},
\end{aligned}
$$

where we have introduced

$$
\begin{aligned}
& \mathbf{r}_{a}=\mathbf{r}_{1}-\mathbf{r}, \\
& \mathbf{r}_{b}=\mathbf{r}_{1}+\mathbf{r},
\end{aligned}
$$

and used that the parity of the function $\phi_{n, l, m}$ with respect to inversion in the origin is $(-1)^{1}$.

By using that ${ }^{24}$

$\sum_{m} Y_{l m}^{*}\left(\theta_{1}, \phi_{1}\right) Y_{l m}\left(\theta_{2}, \phi_{2}\right)=P_{l}\left(\cos \theta_{0}\right) \frac{1}{2 \pi} \sqrt{\frac{2 l+1}{2}}$,

where $\theta_{0}$ is the angle between the directions described by $\left(\theta_{1}, \phi_{1}\right)$ and $\left(\theta_{2}, \phi_{2}\right)$, and $P_{l}$ is the $l$ th Legendre polynomial, we obtain 


$$
\begin{aligned}
\boldsymbol{\epsilon}_{\mathrm{x}}(\mathbf{r})= & 2 \sum_{n_{1}, n_{2}} \sum_{l_{1}, l_{2}} \frac{(-1)^{l_{1}+l_{2}}}{\pi}\left(2 l_{1}+1\right)^{1 / 2}\left(2 l_{2}+1\right)^{1 / 2} \\
& \times \int_{0}^{\infty} \int_{0}^{\pi} R_{n_{1}, l_{1}}^{*}\left(r_{a}\right) R_{n_{2}, l_{2}}\left(r_{a}\right) R_{n_{1}, l_{1}}\left(r_{b}\right) R_{n_{2}, l_{2}}^{*}\left(r_{b}\right) \\
& \times P_{l_{1}}\left(\frac{r_{1}^{2}-r^{2}}{r_{a} r_{b}}\right) P_{l_{2}}\left(\frac{r_{1}^{2}-r^{2}}{r_{a} r_{b}}\right) r_{1} \sin \theta_{1} d \theta_{1} d r_{1} .
\end{aligned}
$$

Here, we have used that

$$
\cos \theta_{0}=\frac{r_{1}^{2}-r^{2}}{r_{a} r_{b}}
$$

due to Eq. (37).

In our calculations we utilize that, due to the spherical symmetry of the atoms, we need only to calculate the energy densities as a function of $r$. For a discrete set of $r$ values for any of the 15 atoms we evaluate the expression of Eq. (39) numerically. Since this easily becomes computationally involved, one has to limit the number of integration points. By varying this number we estimate that our final results for the exchange-energy density are accurate within $1 \%-2 \%$, but the total exchange energy is 1-2 orders of magnitude more accurate.

For the sake of comparison we shall also present results for Coulomb and kinetic-energy densities. For the former we use Eq. (33) with the functions of Eq. (35) in obtaining

$$
\epsilon_{C}(\mathbf{r})=2 \sum_{n_{1}, n_{2}} \sum_{l_{1}, l_{2}} V_{n_{1}, l_{1}}(\mathbf{r}) \rho_{n_{2}, l_{2}}(\mathbf{r}),
$$

where $\rho_{n_{2}, l_{2}}(\mathbf{r})$ is the (spherically) symmetric electron density of the $\left(n_{2}, l_{2}\right)$ shell,

$$
\rho_{n_{2}, l_{2}}(\mathbf{r})=\rho_{n_{2}, l_{2}}(r)=\left|R_{n_{2} l_{2}}(r)\right|^{2} \frac{2 l_{2}+1}{4 \pi}
$$

and $V_{n_{1}, l_{1}}(\mathbf{r})$ is the (also spherically symmetric) Coulomb potential from the electron density $\rho_{n_{1}, l_{1}}(\mathbf{r})$, which obeys Poisson's equation

$$
-\nabla^{2} V_{n_{1}, l_{1}}(\mathbf{r})=4 \pi \rho_{n_{1}, l_{1}}(\mathbf{r}) .
$$

In our calculations we calculate the electron densities of Eq. (42) and solve subsequently (numerically) Poisson's equation (43) for the individual electronic shells.

Finally, the kinetic-energy density, Eq. (34), can in the present case be written as

$$
\begin{aligned}
\epsilon_{\mathrm{k}}(\mathbf{r})= & \sum_{n} \sum_{l} \frac{2 l+1}{8 \pi}\left[-R_{n l}(r) \frac{d^{2} R_{n l}(r)}{d r^{2}}-\frac{2}{r} R_{n l}(r)\right. \\
& \left.\times \frac{d R_{n l}(r)}{d r}+\frac{2 l(l+1)}{r^{2}} R_{n l}^{2}(r)+\left(\frac{d R_{n l}(r)}{d r}\right)^{2}\right],
\end{aligned}
$$

where we have used various well-known properties of the spherical harmonics $Y_{l m}$.

We have calculated these properties together with $|\nabla \rho(\mathbf{r})|$ and $\left|\nabla^{2} \rho(\mathbf{r})\right|$ for the 15 closed-shell atoms mentioned above. As representative examples we show in Fig. 1 the densities for four of those, $\mathrm{He}, \mathrm{Be}, \mathrm{Cd}$, and $\mathrm{Rn}$.

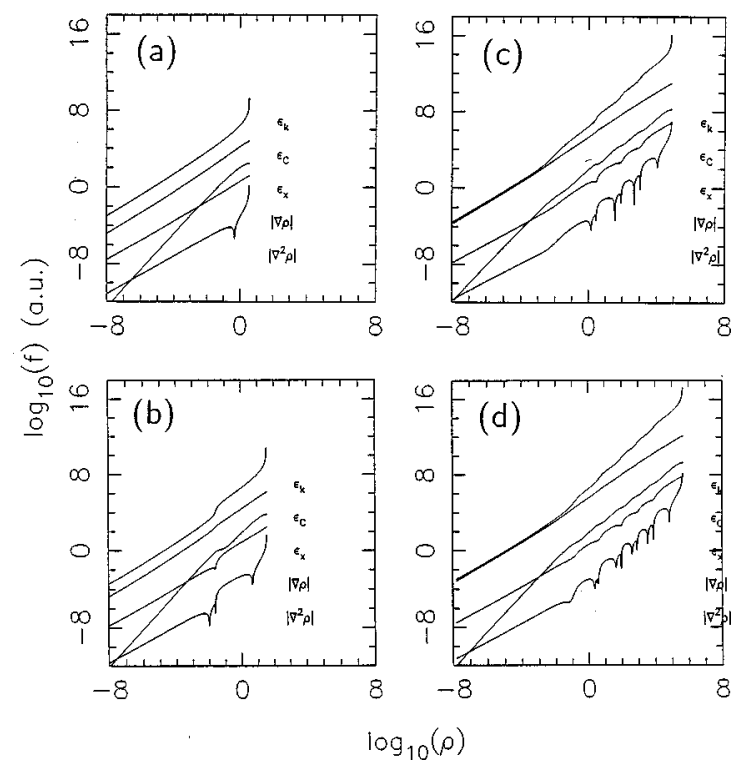

FIG. 1. The exchange-energy density $\epsilon_{\mathrm{x}}$, the kinetic-energy density $\epsilon_{k}$, the Coulomb-energy density $\epsilon_{C},|\nabla \rho|$, and $\left|\nabla^{2} \rho\right|$ for (a) a He atom, (b) a Be atom, (c) a Cd Atom, and (d) a Rn atom. $\epsilon_{k}$ has been multiplied by $10^{6}, \epsilon_{C}$ by $10^{4}, \epsilon_{\mathrm{x}}$ by $10^{2}$, and $\left|\nabla^{2} \rho\right|$ by $10^{-4}$. The various functions are presented as functions of the electron density $\rho$ on a double-logarithmic scale, and the labels on the figure correspond to the order of appearance for $\log \rho=0$.

In Fig. 1 we see that all functions are approximately smooth, monotonous functions of $\rho .|\nabla \rho|$ shows some minor deviations from this behavior which are due to the shell structure. As a further consequence of this, $\left|\nabla^{2} \rho\right|$ has more pronounced oscillations.

In particular the Coulomb-energy density is a very smooth function of $\rho$ which can be explained as being due to the very nonlocal dependence of $\epsilon_{C}$ on the total electron density $\rho(\mathbf{r})$. Also the kinetic-energy density appears as almost straight lines in the plots except for the region absolutely closest to the nuclei (i.e., for the largest values of $\rho$ ), where it raises more steeply. $\epsilon_{\mathrm{k}}$ has, however, some extra features at the positions where $|\nabla \rho|$ has oscillations, i.e., also the kinetic-energy density is sensitive to the shell structure, which should not surprise when considering Eq. (44). It may, on the other hand, be considered more surprising that also the exchange-energy density shows clear shell-structure effects. Thus, although also $\epsilon_{\mathrm{x}}$ is roughly straight lines in Fig. 1 , there are additional oscillations at the positions of the electronic shells. This fact was already recognized in our earlier work. $^{7}$

The exchange and Coulomb-energy densities of Eqs. (39) and (41) appear as double summations over the interactions between pairs of $(n, l)$ shells. In order to study the interactions between the different shells separately, we show in Figs. 2 and 3 the electron densities and the exchange and Coulomb-energy densities for the different shells for the $\mathrm{Ne}$ atom. From Fig. 3 it is clear that the exchange-energy density for intershell interactions [i.e., the $(1 s, 2 s),(1 s, 2 p)$, and $(2 s, 2 p)$ components] are markedly smaller than the corresponding Coulomb-energy densities. This may be taken as a confirmation of the result of Gritsenko et al. ${ }^{25}$ that the exchange potential shows a clear shell structure and that the 

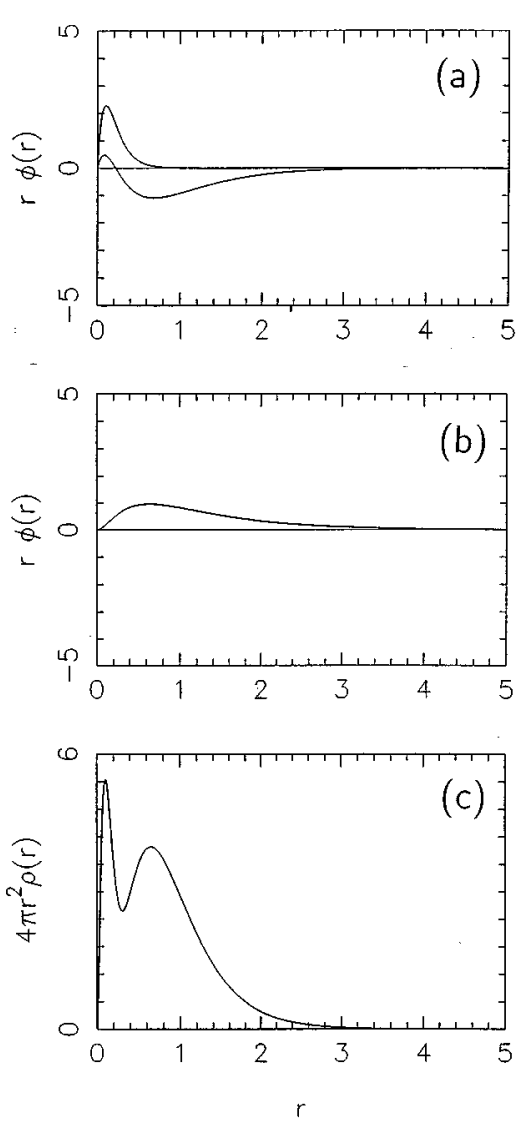

FIG. 2. Orbitals and total electron density for a Ne atom. (a) and (b) show $r R_{n l}(r)$ for (a) $s$ functions and (b) $p$ functions, and (c) shows $4 \pi r^{2} \rho(r)$.

intra-shell interactions are much larger than the intershell interactions. By comparing with Fig. 2 we also see that the structure of the Coulomb-energy density for the electron density of shell $\left(n_{1}, l_{1}\right)$ and the Coulomb potential of shell $\left(n_{2}, l_{2}\right)$ is mainly determined by the structure of the $\left(n_{1}, l_{1}\right)$ shell, manifesting the delocalized nature of the Coulomb potential.

The total exchange- and Coulomb-energy densities for the $\mathrm{Ne}$ atom, obtained by adding the various components of Fig. 3, are shown in Fig. 4. A comparison with Fig. 2 shows clearly the shell structure, first of all for the exchange-energy density. We add that Filippi et al. ${ }^{4}$ also calculated an exchange-energy density for the $\mathrm{Ne}$ atom but obtained by carrying only the $\mathbf{r}_{2}$ integration in Eq. (7) through and defining the resulting function of $\mathbf{r}_{1}$ as the exchange-energy density. Their results show very similar shell structures like those of Fig. 4. This suggests that although our results are obtained for a specific correspondence rule, they are more general. Since we use a different sampling in $\left(\mathbf{r}_{1}, \mathbf{r}_{2}\right)$-space [cf. Eqs. (7), (8), and (32)] this result is far from trivial.

One of the most interesting questions is whether the various energy densities are simple functionals of the electron density. In order to analyze this further we first use that the different energy densities in Fig. 1 appear roughly as straight lines, which also is the case for those atoms not shown; see Fig. 5. We therefore first seek an approximation of the form
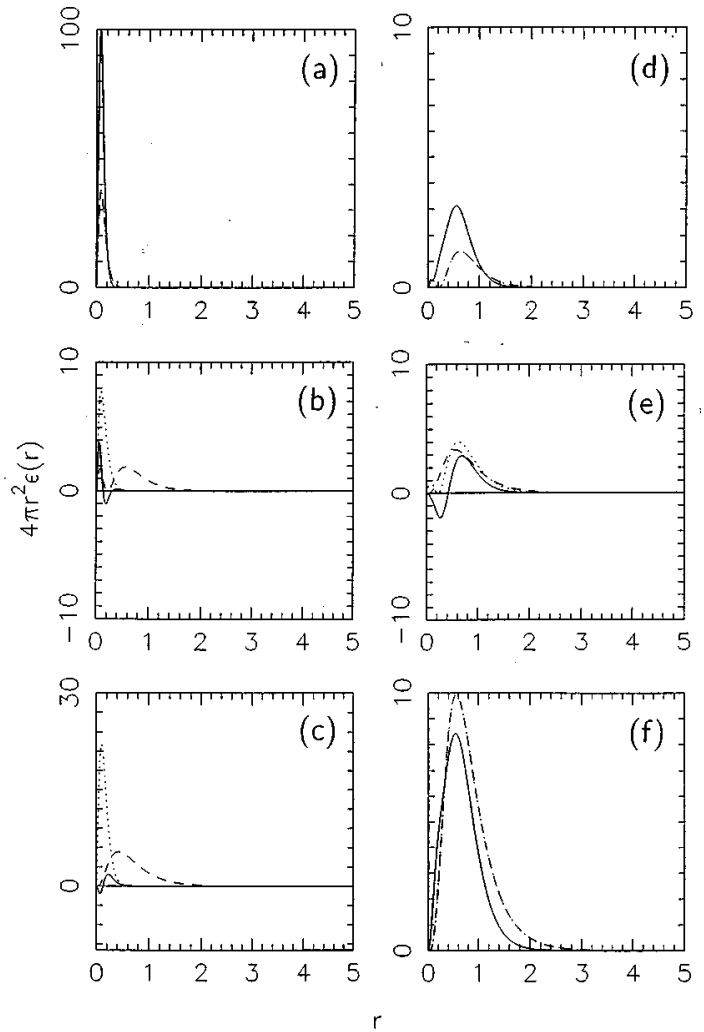

FIG. 3. Exchange- and Coulomb-energy densities for a $\mathrm{Ne}$ atom for the interactions between different pairs of electronic shells $\left(n_{1}, l_{1}\right)$ and $\left(n_{2}, l_{2}\right)$. The full curves show the exchange-energy densities. The dotted curves show the Coulomb-energy density for the electron density of the $\left(n_{1}, l_{1}\right)$ shell in the Coulombic field of the $\left(n_{2}, l_{2}\right)$ shell, whereas the two shells have been interchanged for the dashed curves. The pairs of shells are (a) $(1 s, 1 s)$, (b) $(1 s, 2 s),(\mathrm{c})(1 s, 2 p),(\mathrm{d})(2 s, 2 s),(\mathrm{e})(2 s, 2 p)$, and (f) $(2 p, 2 p)$.

$$
e(r) \simeq \tilde{e}(r)=a \cdot[\rho(r)]^{b},
$$

where $e$ is either $\epsilon_{\mathrm{k}}, \epsilon_{C}, \epsilon_{\mathrm{x}}$, or $|\nabla \rho|$. A least-squares fit for all 15 atoms simultaneously resulted in $(a, b)$ $=(12.038,1.5958),(11.832,1.2071),(0.3072,1.4557)$, and $(6.685,1.2107)$ for the four functions, respectively. We stress that these fits are introduced only as convenient in analyzing our results, but not as accurate representations of those.

Subsequently, we studied renormalized densities obtained by considering the true densities divided by the ap-

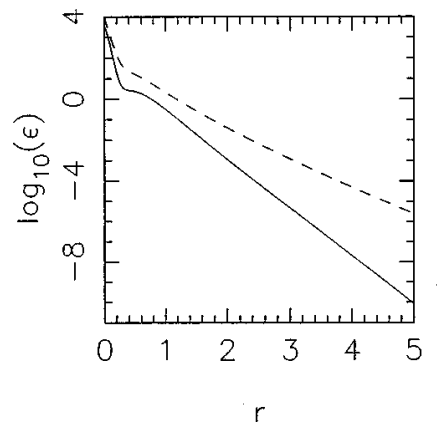

FIG. 4. Exchange (full curve) and Coulomb (dashed curve) energy density for the $\mathrm{Ne}$ atom in a logarithmic scale as a function of $r$. 

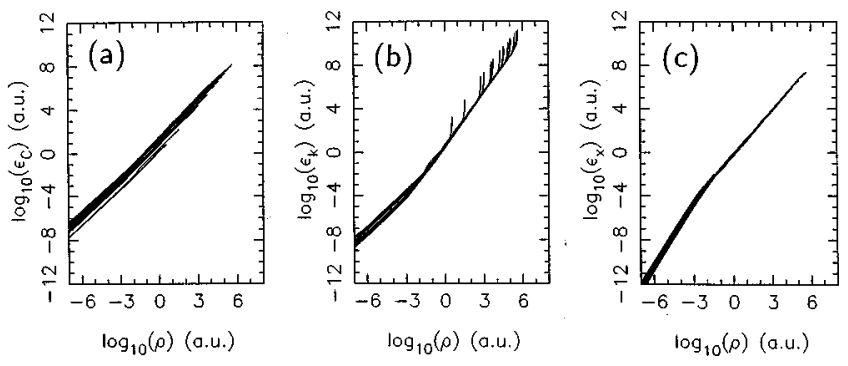

FIG. 5. The (a) Coulomb, (b) kinetic, and (c) exchange-energy density as a function of the electron density for all 15 atoms in double-logarithmic plots.

proximate ones of Eq. (45). These functions are shown in Fig. 6.

From Figs. 5(a) and 6(a) it is seen that the Coulombenergy density is a strongly non-local functional of the electron density. In fact, the 15 curves for the different atoms in Fig. 6(a) are roughly parallel and, for a given $\rho$, the value grows with the total number of electrons of the system of interest.

The kinetic-energy density in Fig. 6(b) is less spread out than the Coulomb-energy density, except for for the largest density for each atom for which the energy density possesses a sharp increase, as well as for the lowest energy density that depends less strongly on the electron density. Both of these features are very clearly recognized in Fig. 6(b). Since the kinetic-energy density involves the first- and second-order derivatives of the basis functions that-being Gaussiansmay have incorrect behaviors for very small and very large $r$, we cannot exclude that these features are of nonphysical origin.

Of the three energy densities in Fig. 5, the exchangeenergy density is the one that shows the smallest variations for a given $\rho$, indicating that this function in fact to a good approximation is a local function of $\rho$. It does, however, show some spread for the smallest values of $\rho$ (i.e., for $\log _{10} \rho \leqslant-2$ ). Therefore, the renormalized energy density of Fig. 6(c) is not a constant. But the fact that $\epsilon_{\mathrm{x}}$ is roughly a constant for a given $\rho$ makes it worthwhile to attempt to compare it with functions of $\rho$ and possibly some of its derivatives. Whether this result is a consequence of the fact that the Weyl correspondence leads to a more local exchange hole than the "standard" definition, ${ }^{20}$ remains an open question.

Finally, it turned out that in order to obtain a uniform description of the energy densities it is more useful to depict
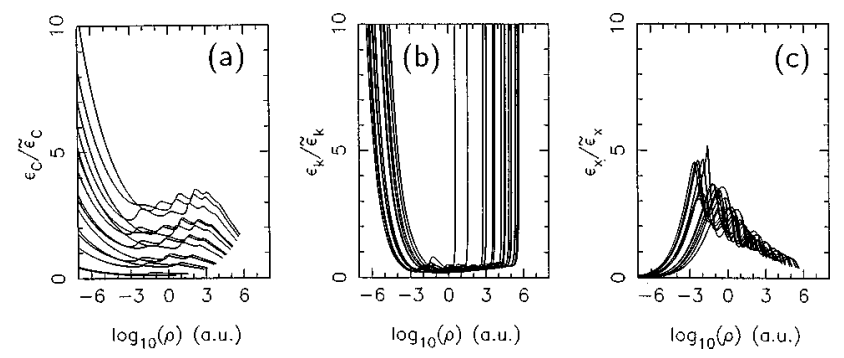

FIG. 6. As Fig. 5, but the renormalized energy densities. them as a function of $\rho$ than as a function of a position-space coordinate.

\section{FACTORIZING $\epsilon_{\boldsymbol{x}}$}

According to the original derivations of Slater ${ }^{26}$ and Gáspár ${ }^{27}$ the exchange-energy density for an extended homogeneous system can be written as

$$
\epsilon_{\mathrm{x} \alpha}=\frac{3}{4}\left(\frac{3}{\pi}\right)^{1 / 3} \rho^{4 / 3} .
$$

This corresponds to $(a, b)=(0.7386,1.3333)$ in Eq. (45), which differs somewhat from the values quoted above obtained through the fit.

The expression of Eq. (46) is the exact one for a homogenous electron gas, so that deviations from this is due to inhomogeneities of the electron density. Accordingly, the function $F$ defined as

$$
\epsilon_{\mathrm{x}}=F \cdot \epsilon_{\mathrm{x} \alpha}
$$

will equal 1 for the homogenous electron gas. In order to include some of the effects of $F$ on inhomogeneities we will here assume that $F$ in any point in position space is a function of $s$ and $t$ defined through

$$
\begin{aligned}
& s=\frac{|\nabla \rho|}{2\left(3 \pi^{2}\right)^{1 / 3} \rho^{4 / 3}}, \\
& t=\frac{\nabla^{2} \rho}{\rho^{5 / 3}} .
\end{aligned}
$$

There exists a number of different proposals for analytical expressions for the function $F(s, t)$ (which in most cases actually is a function of only $s$ ). In order to study how well these perform we show in Fig. 7 the ratios between the approximate exchange-energy densities and our exact one of Fig. 5(c). We shall quantify the performances of the various approximations by considering the quantity

$$
q=\left\{\frac{1}{15} \sum_{i=1}^{15}\left[E_{\mathrm{x}}-4 \pi \int_{0}^{\infty} \tilde{\epsilon}_{\mathrm{x}} r^{2} d r\right]^{2}\right\}^{1 / 2},
$$

where the sum runs over the 15 atoms of our study. $\tilde{\epsilon}_{\mathrm{x}}$ is any of the approximate forms of $\epsilon_{\mathrm{x}}$, and $E_{\mathrm{x}}$ is the exact total exchange energy calculated with the wave functions of Huzinaga and Klobukowski. ${ }^{22} q$ is thus the root of the mean of the squares of the deviations of the total exchange energy. Table I contains the calculated total exchange energies using the different forms to be discussed below for the various atoms of the present study together with the values of $q$. Although related analyses have been published before, we include significantly more (first of all, heavier) atoms and functionals. All atoms are closed-shell atoms but, nevertheless, we can not exclude that in some cases additional correlation effects will modify details of our conclusions.

The homogenous-electron-gas expression of Slater ${ }^{26}$ and Gáspár $^{27}$ [Eq. (46); shown in Fig. 7(a) and given the shorthand label $X \alpha$ in Table I and Fig. 7] is seen to perform reasonably well on the average except for for very low elec- 

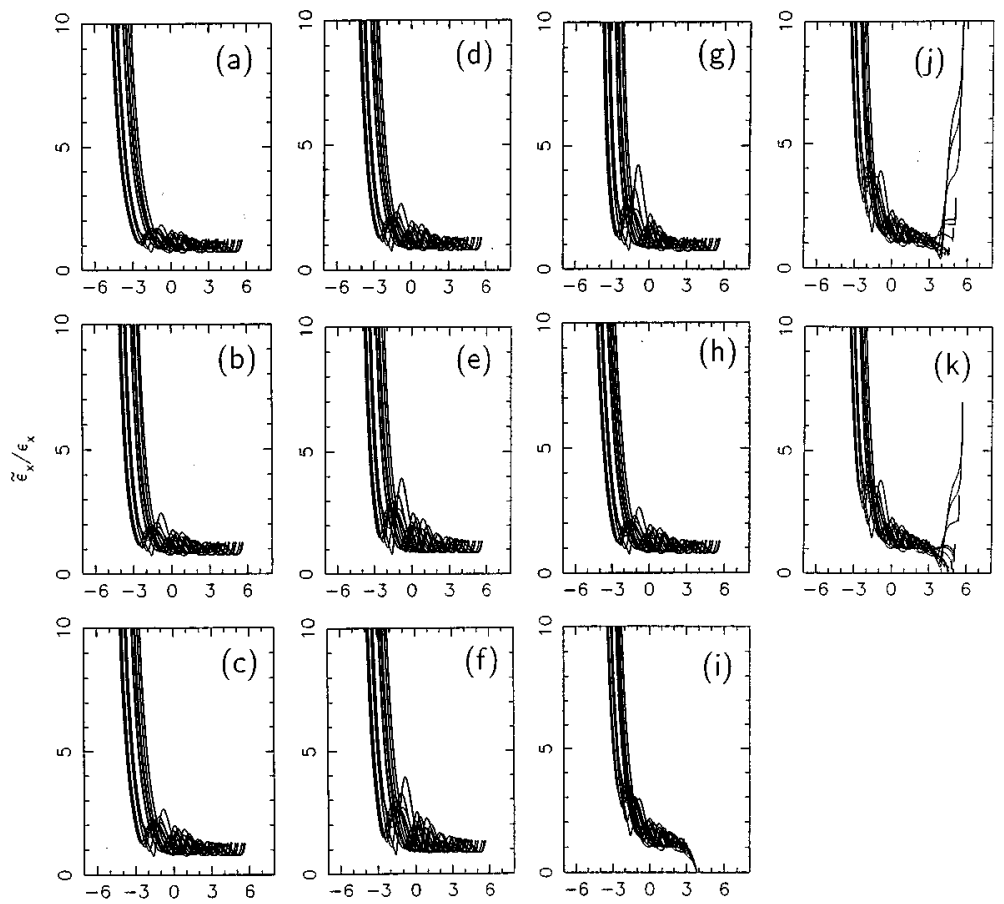

(i)

$\log _{10}(\rho)$ (o.u.)

tron densities, although it does not describe the oscillations due to the electronic shell structure. For this form we find $q=7.30$ a.u.

Sham $^{28}$ (labeled S71 in Table I and Fig. 7) proposed

$F(s, t)=1+0.08641 s^{2}$,

which results in the curves of Fig. 7(b). It does improve the total exchange energies, but, as we shall see later, it grows with $s$ in contrast to our results.

In their first generalized gradient-corrected functional, Perdew and co-workers ${ }^{29}$ suggested using

$$
F(s, t)=\left(1+1.296 s^{2}+14 s^{4}+0.2 s^{6}\right)^{1 / 15},
$$

(labeled PW86) which gives the curves of Fig. 7(c) and a value of $q$ markedly improved compared with that of the $x \alpha$ value. Also this function grows with $s$, but much weaker than that of Sham which is the reason for the worse performance of the latter.

$$
\begin{aligned}
& \text { Becke }^{30} \text { proposed originally } \\
& F(s, t)=1+0.2449 s^{2} \cdot\left(1+0.4254 s^{2}\right)^{-0.8},
\end{aligned}
$$

(labeled B86) giving one of the best values of $q$, i.e., $q$ $=0.19$ a.u., and the curves of Fig. $7(\mathrm{~d})$.

DePristo and Kress ${ }^{31}$ proposed using either

$$
F(s, t)=1+0.2351 s^{2} \frac{1+6.7159 s}{1+2.6913 s^{2}}
$$

(labeled DK87-1) or

$$
F(s, t)=1+0.2351 s^{2} \frac{1+6.4457 s^{0.98}}{1+2.5570 s^{2}},
$$

(labeled DK87-2) which leads to the two sets of curves of Figs. 7(e) and 7(f). As is obvious from these figures, these functionals perform hardly better than the other ones, and, actually, the total exchange energies are worse than those of the $x \alpha$ approximation, $q=11.98$ and 11.97 a.u., respectively.

Becke $^{32}$ has more recently proposed

$F(s, t)=1+0.2743 s^{2}[1+0.1964 s \operatorname{Arsinh}(7.7956 s)]^{-1}$

(labeled B88) for which we in Fig. 7(g) show the resulting curves. Also this gives one of the smallest values of $q, q$ $=0.20$ a.u.

The most recent form of Perdew ${ }^{33}$ has

$F(s, t)=\frac{1+0.1965 s \operatorname{Arsinh}(7.7956 s)+s^{2}\left(0.2743-0.1508 e^{-100 s^{2}}\right)}{1+0.1965 s \operatorname{Arsinh}(7.7956 s)+0.004 s^{4}}$

(labeled P91) whereby the curves of Fig. 7(h) are obtained, and which gives one of the smallest values of $q, q$ $=0.19$ a.u.

All the approximate forms so far have performed reasonably well for larger values of $\rho$, without being able to describe the electronic shell structures well. Only for very small values of $\rho$, these expressions show large deviations from the exact densities. This is different for the expressions of Tozer et al., ${ }^{34}$ according to which

$$
F(s, t)=\sum_{a, c} w_{a c} \rho^{a} s^{c} .
$$

They give three different suggestions (here labeled THG97-1, THG97-2, and THG97-3, respectively) for the spin-unpolarized case, differing in the number of terms (4, 12 , and 12 , respectively) and in the coefficients $w_{a c}$. As seen in Figs. 7(i) $-7(\mathrm{k})$, these forms have also severe deviations for large values of $\rho$, for which they either significantly underestimate or significantly overestimate the exchange- 
TABLE I. The total exchange energies (in a.u.) for the different closed-shell atoms. The headings (a)-(k) correspond to the density-functional expressions of Fig. 7, whereas "present" and "exact" label the values of the present work and the exact total exchange energies, respectively. For the present work we have three different sets differing in the parameters that are set nonzero. These parameters are specified in the heading, too, as are also the short-hand labels for the various approximations. $q$ is the parameter of Eq. (49).

\begin{tabular}{|c|c|c|c|c|c|c|c|c|}
\hline Atom & $\begin{array}{l}\text { (a) } \\
X \alpha\end{array}$ & $\begin{array}{l}\text { (b) } \\
\text { S71 }\end{array}$ & $\begin{array}{c}\text { (c) } \\
\text { PW86 }\end{array}$ & $\begin{array}{l}\text { (d) } \\
\text { B86 }\end{array}$ & $\begin{array}{c}\text { (e) } \\
\text { DK87-1 }\end{array}$ & $\begin{array}{c}(\mathrm{f}) \\
\text { DK87-2 }\end{array}$ & $\begin{array}{l}(\mathrm{g}) \\
\text { B88 }\end{array}$ & \\
\hline $\mathrm{He}$ & 0.88 & 0.97 & 1.03 & 1.02 & 1.26 & 1.26 & 1.01 & \\
\hline $\mathrm{Be}$ & 2.31 & 2.50 & 2.68 & 2.66 & 3.24 & 3.24 & 2.66 & \\
\hline $\mathrm{Ne}$ & 11.03 & 11.55 & 12.22 & 12.15 & 14.04 & 14.04 & 12.14 & \\
\hline $\mathrm{Mg}$ & 14.61 & 15.24 & 16.10 & 16.02 & 18.40 & 18.40 & 16.00 & \\
\hline $\mathrm{Ar}$ & 27.86 & 28.86 & 30.29 & 30.18 & 34.11 & 34.11 & 30.15 & \\
\hline $\mathrm{Ca}$ & 32.59 & 33.71 & 35.34 & 35.22 & 39.69 & 39.69 & 35.19 & \\
\hline $\mathrm{Zn}$ & 65.64 & 67.37 & 69.94 & 69.88 & 77.06 & 77.06 & 69.86 & \\
\hline $\mathrm{Kr}$ & 88.62 & 90.74 & 93.85 & 93.87 & 102.73 & 102.73 & 93.87 & \\
\hline $\mathrm{Sr}$ & 96.36 & 98.60 & 101.91 & 101.96 & 111.38 & 111.38 & 101.96 & \\
\hline $\mathrm{Cd}$ & 141.54 & 144.45 & 148.65 & 148.90 & 161.18 & 161.18 & 148.93 & \\
\hline $\mathrm{Xe}$ & 170.57 & 173.88 & 178.61 & 178.98 & 193.01 & 193.00 & 179.04 & \\
\hline $\mathrm{Ba}$ & 180.24 & 183.68 & 188.60 & 189.02 & 203.63 & 203.61 & 189.08 & \\
\hline $\mathrm{Yb}$ & 265.55 & 269.92 & 275.91 & 276.84 & 295.36 & 295.34 & 276.99 & \\
\hline $\mathrm{Hg}$ & 332.14 & 337.20 & 343.95 & 345.27 & 366.62 & 366.59 & 345.49 & \\
\hline $\mathrm{Rn}$ & 372.98 & 378.46 & 385.67 & 387.23 & 410.29 & 410.26 & 387.49 & \\
\hline$q$ & 7.30 & 4.46 & 0.66 & 0.19 & 11.98 & 11.97 & 0.20 & \\
\hline & (h) & (i) & (j) & (k) & present & present & present & \\
\hline Atom & P91 & THG97-1 & THG97-2 & THG97-3 & $(a, b, f)$ & $(a, b, d, f)$ & $(a, b, c, d, e, f)$ & exact \\
\hline $\mathrm{He}$ & 1.02 & 1.35 & 1.46 & 1.45 & 0.97 & 0.99 & 0.91 & 1.03 \\
\hline $\mathrm{Be}$ & 2.65 & 3.32 & 3.47 & 3.48 & 2.57 & 2.64 & 2.37 & 2.67 \\
\hline $\mathrm{Ne}$ & 12.12 & 14.20 & 14.38 & 14.42 & 12.08 & 12.10 & 12.14 & 12.10 \\
\hline $\mathrm{Mg}$ & 15.98 & 18.47 & 18.68 & 18.73 & 16.01 & 16.02 & 15.86 & 16.00 \\
\hline $\mathrm{Ar}$ & 30.12 & 33.35 & 34.23 & 34.18 & 30.14 & 30.15 & 30.15 & 30.19 \\
\hline $\mathrm{Ca}$ & 35.16 & 38.19 & 39.63 & 39.47 & 35.25 & 35.25 & 35.31 & 35.22 \\
\hline $\mathrm{Zn}$ & 69.83 & 68.25 & 77.40 & 75.65 & 69.86 & 69.82 & 69.78 & 69.65 \\
\hline $\mathrm{Kr}$ & 93.83 & 85.14 & 105.10 & 100.88 & 93.76 & 93.78 & 93.83 & 93.87 \\
\hline $\mathrm{Sr}$ & 101.92 & 89.79 & 114.84 & 109.41 & 101.92 & 101.91 & 101.81 & 101.96 \\
\hline $\mathrm{Cd}$ & 148.88 & 111.59 & 176.55 & 161.08 & 148.89 & 148.87 & 149.01 & 148.94 \\
\hline $\mathrm{Xe}$ & 178.99 & 118.70 & 222.21 & 196.58 & 178.91 & 178.94 & 179.18 & 179.12 \\
\hline $\mathrm{Ba}$ & 189.03 & 119.51 & 238.83 & 208.97 & 189.02 & 189.03 & 189.11 & 189.13 \\
\hline $\mathrm{Yb}$ & 276.93 & 123.90 & 404.47 & 330.02 & 276.85 & 276.85 & 276.29 & 276.26 \\
\hline $\mathrm{Hg}$ & 345.43 & 106.18 & 569.14 & 442.10 & 345.38 & 345.36 & 345.33 & 345.37 \\
\hline $\mathrm{Rn}$ & 387.42 & 84.07 & 689.47 & 520.47 & 387.32 & 387.33 & 387.58 & 387.58 \\
\hline$q$ & 0.19 & 110.36 & 104.29 & 45.42 & 0.19 & 0.19 & 0.11 & \\
\hline
\end{tabular}

energy density. Accordingly, the $q$ values for these are large, i.e., $q=110.36,104.29$, and 45.42 a.u. for the three forms, respectively.

From the results of Fig. 7 as well as the values of $q$ for the different approximate functionals it is obvious that none of them provides an excellent fit for the exchange-energy density, and in fact when basing the discussion on $q$ of Eq. (49), the two forms of Becke ${ }^{30}$ and Perdew ${ }^{33}$ of Eqs. (52) and (56), respectively, are the best ones. In order to see how this becomes the case we show in Figs. 8(a) and 8(b) our calculated $F$ based on Eq. (47). It is seen that in particular for small $s, F$ is not unique, which implies that it is not possible to describe $F$ exactly when considering only a dependence on $s$ (a similar result was recently found also by Jemmer and Knowles, ${ }^{35}$ but from very different arguments). But in addition we see that $F \rightarrow 0$ for large $s$, and of the forms above this is only the case for the functional of Perdew of Eq. (56). In Fig. 8(a) we see also that $F$ approaches the value 1 for $s$ $\rightarrow 0$, as it should for the homogenous electron gas. Finally, we add that the smallest values of $s$ correspond to the largest values of $\rho$.

\section{AN ALTERNATIVE FUNCTIONAL}

It is obvious from Figs. 8(a) and 8(b) that $F$ is not a unique function of $s$. By plotting $F$ as a function of $t$ [Eq. (48)] we obtain the results of Figs. 8(c)-8(e), from which it is clear that also $t$ is not able to specify $F$ uniquely. Similar to the dependence of $F$ on $s$, also for large $t, F \rightarrow 0$, and small $t$ corresponds to large $\rho$.

We shall therefore attempt to write $F$ as a function of both $s$ and $t$. However, it turns out that $F$ is not uniquely specified through $s$ and $t$ either. Moreover, our study does not at all cover the complete $(s, t)$ plane, but, instead, $s$ and $t$ are somewhat interrelated.

Despite these problems we seek a simple function of $s$ and $t$ that describes the results of Fig. 8. Since our exchange hole can be argued to be localized, ${ }^{20}$ our results form an 

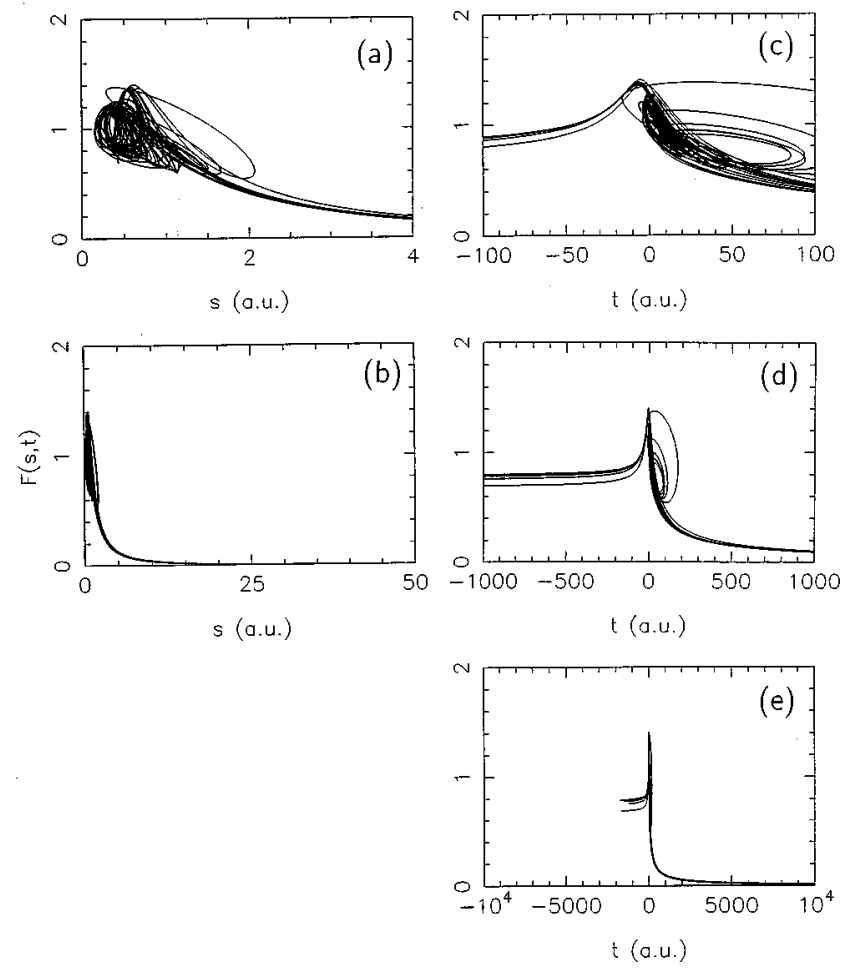

FIG. 8. Each panel shows the superposition of the 15 curves (one for each atom) obtained by plotting $F$ of Eq. (47) as a function [(a),(b)] of $s$ and [(c)-(e)] of $t$. (a) and (b) [(c), (d), and (e)] differ by the abscissa scales.

excellent starting point for studying a local description of exchange effects. From Fig. 8 we see that $F$ has to approach 0 for $s$ or $t$ being large (notice, that our study does not cover the case that $t$ is large but negative), and since $F$ was found to be $>0$ everywhere in our calculations (which is a far from obvious finding), and since $F \equiv 1$ for $s=t=0$, we approximate $F$ as

$$
F(s, t) \simeq \frac{1}{1-f^{2}+\left(f+a s+b t+c s^{2}+d s t+e t^{2}\right)^{2}} .
$$

The constants $f, a, b, c, d$, and $e$ were determined through a least-squares fit to our results. We considered three different approximations differing in the parameters that were allowed to be nonzero. Our optimized parameters from the three set of calculations are

$$
\begin{aligned}
& f=0.69360,0.77907,0.44157, \\
& a=-0.025964,-0.017162,-0.25294, \\
& b=-0.0074335,-0.0083317,0.035977, \\
& c=0.0,0.0,0.041285, \\
& d=0.0,0.0018606,-0.023244, \\
& e=0.0,0.0,-0.00063501,
\end{aligned}
$$

where the first, second, and third set was obtained by optimizing $(a, b, f),(a, b, d, f)$, and $(a, b, c, d, e, f)$, respectively, and keeping all other parameters fixed at 0 . The value of $q$ of Eq. (49) becomes $0.19,0.19$, and 0.11 a.u., respectively, i.e., up to about $40 \%$ smaller than that of any of the other ap-

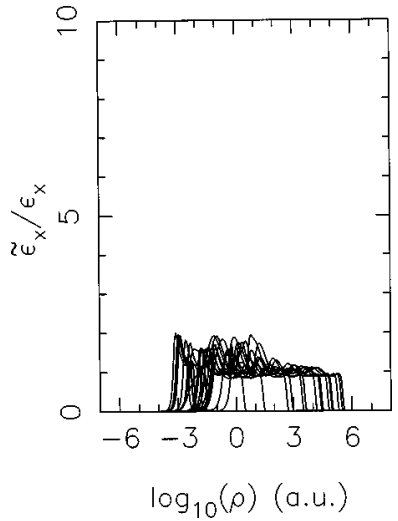

FIG. 9. As Fig. 7, but for the approximate form of Eqs. (58) and (59) using all six fit parameters.

proximate functionals. However, using just the total exchange energy as a measure for the quality of the approximation gives that the increased number of parameters hardly improves the fit. Moreover, the most complicated fit appears to improve the description of the more complicated systems (i.e., the heavier atoms) but simultaneously reducing the quality for the lighter atoms.

The quality of the approximate expression of Eqs. (58) and (59) is seen in Fig. 9. In fact, only in the absolutely outer- or innermost parts of the atoms, does the ratio between the exact and the approximate exchange-energy density show significant deviations from the ideal value of 1 . This indicates that this form is much better capable of describing the oscillatory behavior of the exchange-energy density than any of the previously proposed forms. We add that our approximate forms with a smaller number of parameters lead to similar results except in the low- $\rho$ limit.

\section{CONCLUSIONS}

In the present work we have used the concept of phasespace functions to define densities in position space for arbitrary quantum-mechanical operators and arbitrary states. The phase-space functions are intimately related to the correspondence between classical functions and quantum mechanical operators. Therefore, the fact that more different correspondence rules are possible leads to different phase-space functions. However, the Wigner phase-space function, which follows from the Weyl correspondence rule, has several appealing properties compared with any other phase-space function. Therefore, we have here focused on this, whereby the position-space densities for arbitrary operators and states are uniquely determined.

We concentrated on the quantum-mechanical exchangeenergy operator for which we defined a position-space density for any $\mathrm{N}$-electron state that could be written as a single Slater determinant of $N / 2$ doubly occupied orbitals. We stressed that the resulting exchange potential, as derived within the concept of density-functional theory, is independent of the chosen correspondence rule. This is important since it assures that the orbitals will be independent of the correspondence rule. 
Subsequently we calculated the exchange-energy density for a number of closed-shell atoms and compared it with Coulomb and exchange-energy densities. We found that of the three, the exchange-energy density in a given point $\mathbf{r}$ shows the weakest dependence on the electron density in points far away from $\mathbf{r}$, although effects due to the electronic shells were recognizable. By separating the exchange-energy density into various inter- and intrashell components we found that the interactions between different $(n, l)$ shells were markedly smaller than the intrashell components, once again supporting the local nature of the exchange-energy density.

Since our results provide a very detailed description of exchange effects and since it can be argued that the Weyl correspondence leads to a local exchange hole, ${ }^{20}$ we could access the quality of various approximate local descriptions of the exchange-energy density, much more detailed than usually is the case. We found that none of the existing approximate schemes describe the details of the exchangeenergy densities accurately, and in particular the regions closest to the nuclei and most far from those were poorly described. As a consequence we proposed an alternative approximate form. To this end we found that it was useful to consider a functional that depends not only on $|\nabla \rho|$ but also on $\nabla^{2} \rho$. The functional is given in Eqs. (58) and (59) but is only applicable for the spin-unpolarized case. We found that the new form improves the total exchange energies with about $40 \%$, but, what may be more important when studying the formation of chemical bonds, it provided a more accurate description of the oscillatory behavior of the exchangeenergy density. It will be useful to extend our form to the spin-polarized case as well as to try its performance on various smaller molecules. Results on such studies will be reported elsewhere.

\section{ACKNOWLEDGMENTS}

This work was supported by the Deutsche Akademische Austausch Dienst (DAAD) and by the Danish Natural Science Research Council. One of the authors (M.S.) is grateful to Fonds der Chemischen Industrie for very generous support.
${ }^{1}$ Density-Functional Methods in Chemistry and Materials Science, edited by M. Springborg (Wiley, Chichester, 1997).

${ }^{2}$ P. Hohenberg and W. Kohn, Phys. Rev. 136, B864 (1964).

${ }^{3}$ W. Kohn and L. J. Sham, Phys. Rev. 140, A1133 (1965).

${ }^{4}$ C. Filippi, X. Gonze, and C. J. Umrigar, in Recent Developments and Applications of Density Functional Theory, edited by J. M. Seminario (Elsevier, Amsterdam, 1996).

${ }^{5}$ O. V. Gritsenko, R. van Leeuwen, and E. J. Baerends, J. Chem. Phys. 104, 8535 (1996).

${ }^{6}$ R. Q. Hood, M. Y. Chou, A. J. Williamson, G. Rajagopal, R. J. Needs, and W. M. C. Foulkes, Phys. Rev. Lett. 78, 3350 (1997).

${ }^{7}$ M. Springborg and J. P. Dahl, in Local Density Approximations in Quantum Chemistry and Solid State Physics, edited by J. P. Dahl and J. Avery (Plenum, New York, 1984).

${ }^{8}$ S. K. Ghosh and R. G. Parr, Phys. Rev. A 34, 785 (1986).

${ }^{9}$ K. Burke and J. P. Perdew, Int. J. Quantum Chem. 56, 199 (1995).

${ }^{10}$ A. Zupan, K. Burke, M. Ernzerhof, and J. P. Perdew, J. Chem. Phys. 106, 10184 (1997).

${ }^{11}$ J. P. Perdew, M. Ernzerhof, A. Zupan, and K. Burke, J. Chem. Phys. 108, 1522 (1998)

${ }^{12}$ F. G. Cruz, K.-C. Lam, and K. Burke, J. Phys. Chem. A 102, 4911 (1998).

${ }^{13}$ K. Burke, F. G. Cruz, and K.-C. Lam, J. Chem. Phys. 109, 8161 (1998).

${ }^{14}$ L. Cohen, J. Math. Phys. 7, 781 (1966).

${ }^{15}$ M. Springborg, J. Phys. A 16, 535 (1983).

${ }^{16}$ J. P. Dahl, Theor. Chim. Acta 81, 319 (1992).

${ }^{17}$ J. P. Dahl, Phys. Scr. 25, 499 (1982).

${ }^{18}$ E. P. Wigner, Phys. Rev. 40, 749 (1932).

${ }^{19}$ J. P. Dahl, in Energy Storage and Redistribution in Molecules, edited by J. Hinze (Plenum, New York, 1995).

${ }^{20}$ M. Springborg and J. P. Dahl (unpublished).

${ }^{21}$ J. P. Dahl and B. Amstrup, J. Mol. Struct.: THEOCHEM 261, 345 (1992).

${ }^{22}$ S. Huzinaga and M. Klobukowski, Chem. Phys. Lett. 212, 260 (1993).

${ }^{23}$ U. von Barth and L. Hedin, J. Phys. C 5, 1629 (1972).

${ }^{24}$ J. D. Talman, Special Functions: A Group Theoretical Approach (Benjamin, New York, 1968).

${ }^{25}$ O. Gritsenko, R. van Leeuwen, and E. J. Baerends, J. Chem. Phys. 101, 8955 (1994)

${ }^{26}$ J. C. Slater, Phys. Rev. 81, 385 (1951).

${ }^{27}$ R. Gáspár, Acta Phys. Acad. Sci. Hung. 3, 263 (1954).

${ }^{28}$ L. J. Sham, in Computational Methods in Band Theory, edited by P. Marcus, J. F. Janak, and A. R. Williams (Plenum, New York, 1971).

${ }^{29}$ J. Perdew and Wang Yue, Phys. Rev. B 33, 8800 (1986).

${ }^{30}$ A. D. Becke, J. Chem. Phys. 85, 7184 (1986).

${ }^{31}$ A. E. DePristo and J. R. Kress, J. Chem. Phys. 86, 1425 (1987).

${ }^{32}$ A. D. Becke, Phys. Rev. A 38, 3098 (1988).

${ }^{33}$ J. Perdew, in Electronic Structure of Solids, edited by P. Ziesche and H. Eschrig (Akademie, Berlin, 1991).

${ }^{34}$ D. J. Tozer, N. C. Handy, and W. H. Green, Chem. Phys. Lett. 273, 183 (1997).

${ }^{35}$ P. Jemmer and P. J. Knowles, Phys. Rev. A 51, 3571 (1995). 